Special Issue: 25 years of the Mizar Mathematical Library

FORMALIZED MATHEMATICS

Vol. 22, No. 2, Pages 99-103, 2014

DE GRUYTER

OPEN

DOI: $10.2478 /$ forma-2014-0011

degruyter.com/view/j/forma

\title{
Pseudo-Canonical Formulae are Classical
}

\author{
Marco B. Caminati ${ }^{1}$ \\ School of Computer Science \\ University of Birmingham \\ Birmingham, B15 2TT \\ United Kingdom
}

\author{
Artur Korniłowicz \\ Institute of Informatics \\ University of Białystok \\ Sosnowa 64, 15-887 Białystok \\ Poland
}

Summary. An original result about Hilbert Positive Propositional Calculus introduced in [11 is proven. That is, it is shown that the pseudo-canonical formulae of that calculus (and hence also the canonical ones, see [17]) are a subset of the classical tautologies.

MSC: 03B20 03B35

Keywords: Hilbert positive propositional calculus; classical logic; canonical formulae

MML identifier: HILBERT4, version: 8.1.03 5.23.1207

The notation and terminology used in this paper have been introduced in the following articles: [13], [1, [14], [10], [9], [15], [3], 44, [5], [6], 11], [16], [17, [2], [7, [18, [20], 22], 21], 12], [19], and [8].

\section{Preliminaries about Injectivity, Involutiveness, Fixed Points}

From now on $a, b, c, x, y, z, A, B, C, X, Y$ denote sets, $f, g$ denote functions, $V$ denotes a SetValuation, $P$ denotes a permutation of $V, p, q, r, s$ denote elements of HP-WFF, and $n$ denotes an element of $\mathbb{N}$.

Let us consider $X$ and $Y$. Let $f$ be a relation between $X$ and $Y$. Note that $\operatorname{id}_{X} \cdot f$ reduces to $f$ and $f \cdot \operatorname{id}_{Y}$ reduces to $f$.

Now we state the proposition:

(1) Let us consider one-to-one functions $f, g$. If $f^{-1}=g^{-1}$, then $f=g$.

\footnotetext{
${ }^{1}$ My work has been partially supported by EPSRC grant EP/J007498/1 and an LMS Computer Science Small Grant. 
One can verify that there exists a function which is involutive.

Let us consider $A$. Let us observe that there exists a permutation of $A$ which is involutive.

Now we state the propositions:

(2) Let us consider an involutive function $f$. Suppose $\operatorname{rng} f \subseteq \operatorname{dom} f$. Then $f \cdot f=\operatorname{id}_{\operatorname{dom} f}$.

(3) Let us consider a function $f$. If $f \cdot f=\operatorname{id}_{\operatorname{dom} f}$, then $f$ is involutive.

(4) Let us consider an involutive function $f$ from $A$ into $A$. Then $f \cdot f=\operatorname{id}_{A}$. The theorem is a consequence of (2).

(5) Let us consider a function $f$ from $A$ into $A$. If $f \cdot f=\operatorname{id}_{A}$, then $f$ is involutive. The theorem is a consequence of (3).

Observe that every function which is involutive is also one-to-one.

Let us consider $A$. Let $f$ be an involutive permutation of $A$. One can verify that $f^{-1}$ is involutive.

Let $n$ be a non zero natural number. Observe that $[0 \longmapsto n, n \longmapsto 0]$ is without fixpoints.

Let $z$ be a zero natural number. Note that fixpoints $[z \longmapsto n, n \longmapsto z]$ is empty.

Let $X$ be a non empty set. Observe that there exists a permutation of $X$ which is non empty and involutive.

Let us consider $A$ and $B$. Let $f$ be an involutive function from $A$ into $A$ and $g$ be an involutive function from $B$ into $B$. Observe that $f \times g$ is involutive.

Let $A, B$ be non empty sets, $f$ be an involutive permutation of $A$, and $g$ be an involutive permutation of $B$. Observe that $f \Rightarrow g$ is involutive.

\section{Facts about Perm's Fixed Points}

Now we state the propositions:

(6) If $x$ is a fixpoint of $\operatorname{Perm}(P, q)$, then $\operatorname{SetVal}(V, p) \longmapsto x$ is a fixpoint of $\operatorname{Perm}(P, p \Rightarrow q)$.

(7) If $\operatorname{Perm}(P, q)$ has fixpoints, then $\operatorname{Perm}(P, p \Rightarrow q)$ has fixpoints. The theorem is a consequence of $(6)$.

(8) If $\operatorname{Perm}(P, p)$ has fixpoints and $\operatorname{Perm}(P, q)$ is without fixpoints, then $\operatorname{Perm}(P, p \Rightarrow q)$ is without fixpoints.

\section{Axiom of Choice in Functional Form via the Fraenkel Operator}

Let $X$ be a set. The functor ChoiceOn $X$ yielding a set is defined by the term

(Def. 1) $\quad\{\langle x$, the element of $x\rangle$, where $x$ is an element of $X \backslash\{\emptyset\}: x \in X \backslash\{\emptyset\}\}$. 
One can check that ChoiceOn $X$ is relation-like and function-like.

Let us consider $f$. The functor FieldCover $f$ yielding a set is defined by the term

(Def. 2) $\{\{x, f(x)\}$, where $x$ is an element of $\operatorname{dom} f: x \in \operatorname{dom} f\}$.

The functor SomePoints $f$ yielding a set is defined by the term

(Def. 3) field $f \backslash$ rng ChoiceOn FieldCover $f$.

The functor OtherPoints $f$ yielding a set is defined by the term

(Def. 4) (field $f \backslash$ fixpoints $f$ ) \SomePoints $f$.

Let us consider $g$. Let us observe that OtherPoints $g \cap$ SomePoints $g$ is empty.

\section{Building a Suitable Set Valuation and a Suitable Permutation OF IT}

Let us consider $x$. The functor $\operatorname{ToHilb}(x)$ yielding a set is defined by the term

(Def. 5) $\quad\left(\operatorname{id}_{1}+\cdot\left(1 \times \emptyset^{x}\right) \cdot\left(\emptyset^{x} \times\{1\}\right)\right)+\cdot\left(\{1\} \times \emptyset^{x}\right) \cdot\left(\emptyset^{x} \times\{0\}\right)$.

Note that $\operatorname{ToHilb}(x)$ is function-like and relation-like.

Now we state the propositions:

(9) If $x \neq \emptyset$, then $\operatorname{ToHilb}(x)=\mathrm{id}_{1}$.

(10) $\operatorname{ToHilb}(\emptyset)=[0 \longmapsto 1,1 \longmapsto 0]$.

Let $v$ be a function. The functor ToHilbPerm $(v)$ yielding a set is defined by the term

(Def. 6) the set of all $\langle n, \operatorname{ToHilb}(v(n))\rangle$ where $n$ is an element of $\mathbb{N}$.

The functor ToHilbVal $(v)$ yielding a set is defined by the term

(Def. 7) the set of all $\langle n$, dom ToHilb $(v(n))\rangle$ where $n$ is an element of $\mathbb{N}$.

One can check that ToHilbVal $(v)$ is function-like and relation-like and ToHilbPerm $(v)$ is function-like and relation-like and $\operatorname{ToHilbVal}(v)$ is $\mathbb{N}$-defined and $\operatorname{ToHilbVal}(v)$ is total and ToHilbPerm $(v)$ is $\mathbb{N}$-defined and $\operatorname{ToHilbPerm}(v)$ is total.

One can verify that $\operatorname{ToHilbVal}(v)$ is non-empty.

Let us consider $x$. Let us note that $\operatorname{ToHilb}(x)$ is symmetric.

Let $v$ be a function. Observe that the functor ToHilbPerm $(v)$ yields a permutation of $\operatorname{ToHilbVal}(v)$.

A set valuation is a many sorted set indexed by $\mathbb{N}$. From now on $v$ denotes a set valuation.

Let us consider $p$ and $v$. Note that $\operatorname{Perm}(\operatorname{ToHilbPerm}(v), p)$ is involutive. 


\section{Classical Semantics via SetValo, an Extension of SetVal}

Let $V$ be a set valuation. The functor $\operatorname{SetVal}_{0} V$ yielding a many sorted set indexed by HP-WFF is defined by

(Def. 8) (i) $i t($ VERUM $)=1$, and

(ii) for every $n, i t(\operatorname{prop} n)=V(n)$, and

(iii) for every $p$ and $q$, it $(p \wedge q)=i t(p) \times i t(q)$ and $i t(p \Rightarrow q)=(i t(q))^{i t(p)}$.

Let us consider $v$ and $p$. The functor $\operatorname{SetVal}_{0}(v, p)$ yielding a set is defined by the term

(Def. 9) $\left(\operatorname{SetVal}_{0} v\right)(p)$.

We say that $p$ is classical if and only if

(Def. 10) $\operatorname{SetVal}_{0}(v, p) \neq \emptyset$.

One can check that every element of HP-WFF which is pseudo-canonical is also classical.

Let us consider $v$. Let $p$ be a classical element of HP-WFF. Note that $\operatorname{SetVal}_{0}(v, p)$ is non empty.

\section{REFERENCES}

[1] Grzegorz Bancerek. Cardinal numbers Formalized Mathematics, 1(2):377-382, 1990.

[2] Grzegorz Bancerek. The ordinal numbers. Formalized Mathematics, 1(1):91-96, 1990.

[3] Czesław Byliński. Functions and their basic properties Formalized Mathematics, 1(1): 55-65, 1990.

[4] Czesław Byliński. Functions from a set to a set Formalized Mathematics, 1(1):153-164, 1990.

[5] Czesław Byliński. Basic functions and operations on functions. Formalized Mathematics, 1(1):245-254, 1990.

[6] Czesław Byliński. The modification of a function by a function and the iteration of the composition of a function. Formalızed Mathematıcs, 1(3):521-527, 1990.

[7] Czesław Byliński. Partial functions Formalized Mathematics, 1(2):357-367, 1990.

[8] Czesław Byliński. Some basic properties of sets Formalized Mathematics, 1(1):47-53, 1990.

[9] Marco B. Caminati. Preliminaries to classical first order model theory. Formalized Mathematics, 19(3):155-167, 2011. doi 10.2478/v10037-011-0025-2

[10] Agata Darmochwał. Finite sets. Formalized Mathematics, 1(1):165-167, 1990.

[11] Adam Grabowski. Hilbert positive propositional calculus Formalized Mathematics, 8(1): 69-72, 1999.

[12] Beata Padlewska. Families of sets Formalized Mathematics, 1(1):147-152, 1990.

[13] Piotr Rudnicki and Andrzej Trybulec. Abian's fixed point theorem. Formalized Mathematics, 6(3):335-338, 1997.

[14] Andrzej Trybulec. Domains and their Cartesian products Formalized Mathematics, 1(1): $115-122,1990$.

[15] Andrzej Trybulec. Binary operations applied to functions Formalized Mathematics, 1 (2):329-334, 1990.

[16] Andrzej Trybulec. Defining by structural induction in the positive propositional language Formalized Mathematıcs, 8(1):133-137, 1999.

[17] Andrzej Trybulec. The canonical formulae. Formalized Mathematics, 9(3):441-447, 2001.

[18] Andrzej Trybulec. Classes of independent partitions Formalized Mathematics, 9(3): 623-625, 2001. 
[19] Zinaida Trybulec. Properties of subsets. Formalized Mathematics, 1(1):67-71, 1990.

[20] Edmund Woronowicz. Relations and their basic properties Formalized Mathematics, 1 (1):73-83, 1990.

[21] Edmund Woronowicz. Relations defined on sets Formalized Mathematics, 1(1):181-186, 1990.

[22] Edmund Woronowicz and Anna Zalewska. Properties of binary relations. Formalized Mathematics, 1(1):85-89, 1990.

Received May 25, 2014 